\title{
APPLiCATIONS OF FLOATING-GATE MOSFET IN THE DESIGN OF INVERTER AND RING OSCILLATOR
}

\author{
Roshani Gupta, Rockey Gupta and Susheel Sharma
}

Department of Physics and Electronics, University of Jammu, Jammu-180006, India

\begin{abstract}
This paper presents the application of floating-gate MOSFET (FGMOS) in the design of low voltage and high speed digital circuits wherein threshold voltage tunability of FGMOS has been exploited to enhance the performance of inverter in terms of various parameters like switching threshold voltage, noise margins, propagation delay and energy delay product. It has been observed that by varying the bias voltage in FGMOS, the voltage transfer characteristics can be altered that result in lowering of switching threshold voltage, increased noise margins, reduced propagation delay and less energy delay product as compared to the standard CMOS inverter. This paper also demonstrates the design of ring oscillator using FGMOS and it has been found that FGMOS based ring oscillator exhibits higher frequency of oscillation as compared to its CMOS counterpart. The performance of these circuits has been verified through PSpice simulations carried out using level 7 parameters in $0.13 \mu \mathrm{m}$ CMOS technology with a supply voltage of $1 \mathrm{~V}$.
\end{abstract}

\section{KEYWORDS}

Floating-gate MOSFET, inverter, voltage transfer characteristics, propagation delay, energy delay product, ring oscillator

\section{INTRODUCTION}

The design of digital integrated circuits with very low power consumption and without much degradation in speed has always been the focal point of researchers particularly in sub-micron regime [1-3]. Since there is always a trade-off between power dissipation and time delay, therefore, reducing the power dissipation and still maintaining the appreciable performance in terms of operating speed is much desirable. With the immense demand of portable and battery driven applications, there is a need for new and alternative circuit design techniques to implement high performance and low power digital circuits. Further, with reducing feature size of devices, the lowering of operating supply voltage is obvious but at the expense of speed. Hence, for optimum performance of digital circuits, alternative design techniques should be explored [4-6].

CMOS inverter forms a basic building block of digital sub-circuits in mixed mode circuits with limitation of high switching threshold voltage resulting in degraded performance. Floating-gate MOSFET (FGMOS) has been widely used in the design of low voltage analog and digital circuits due to its unique characteristic of threshold voltage tuning with a bias voltage, thus imparting enhancement in performance. In this paper, we have employed floating-gate MOSFET (FGMOS) to design an inverter which has been further used to implement a ring oscillator. The paper has been divided in various sections briefly introducing FGMOS, its application in the design of inverter and ring oscillator. The performance of the designed circuits has been found to be enhanced vis-à-vis their conventional CMOS versions. The workability of these circuits has been

DOI : 10.14810/elelij.2016.5204 
verified through PSpice simulations carried out using level 7 parameters in $0.13 \mu \mathrm{m}$ CMOS technology with a supply voltage of $1 \mathrm{~V}$.

\section{FloATING-GATE MOS TRANSISTOR}

The Floating-Gate MOS transistor (FGMOS) is basically a modified form of simple MOSFET where extra capacitances have been introduced between the conventional gate and the multi-input signal gates. By applying a bias voltage on one of the input gates, the threshold voltage of FGMOS can be reduced. A number of secondary gates or input terminals are deposited above the floating-gate (FG) which are electrically isolated from it but capacitively connected to it. Since FG is completely surrounded by highly resistive material, so for dc operation, FG acts as floating node. Programming of the FGMOS introduces a charge on its floating-gate that shifts the threshold voltage and thus, provides a control over the device functionality [7-10]. The equivalent schematic for an N-input and n-channel FGMOS is shown in Fig. 1 [11].

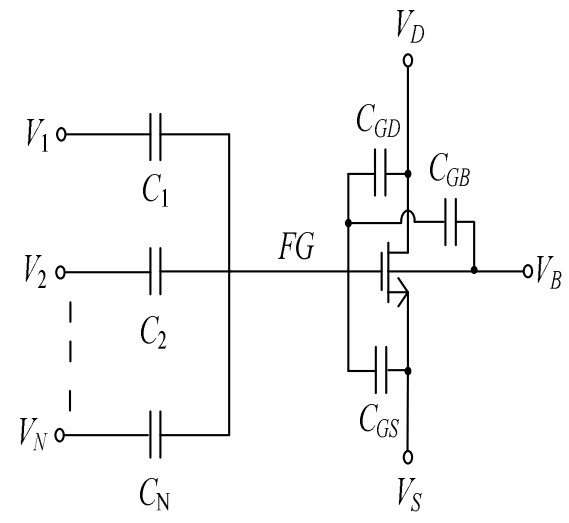

Figure 1. Floating-gate MOSFET

In a two-input n-channel FGMOS, input voltage $\left(V_{i n}\right)$ is applied through $C_{1}$ and bias voltage $\left(V_{\text {bias }}\right)$ is applied through $C_{2}$ which provides tunability to the conventional threshold voltage $\left(V_{T}\right)$ of the FGMOS and $V_{T}$ adjusts to a new value $V_{T, \text { eff }}$ given as [12]:

$$
V_{T, e f f}=\frac{V_{T}-V_{\text {bias }} k_{2}}{k_{1}}
$$

where $k_{1}=\frac{C_{1}}{C_{T}}$ and $k_{2}=\frac{C_{2}}{C_{T}}$ and $C_{1}$ and $C_{2}$ are the capacitances between floating-gate and control gates and $C_{T}=C_{1}+C_{2}+C_{G S}+C_{G D}+C_{G B}$. We observe that $V_{T, e f f}$ will be less than $V_{T}$ if we select $V_{\text {bias }}>V_{T}$ and $k_{2}>k_{1}$, implying $C_{2}>C_{1}$. Thus, in FGMOS we can select $V_{T \text {, eff }}$ lower than normal $V_{T}$.

Now, by selecting $W / L$ of FGMOS as $1.3 \mu \mathrm{m} / 0.13 \mu \mathrm{m}$ and with supply voltage of $1 \mathrm{~V}$, the drain and transfer characteristics are shown in Figs. 2 and 3 respectively. 
Electrical and Electronics Engineering: An International Journal (ELELIJ) Vol 5, No 2, May 2016
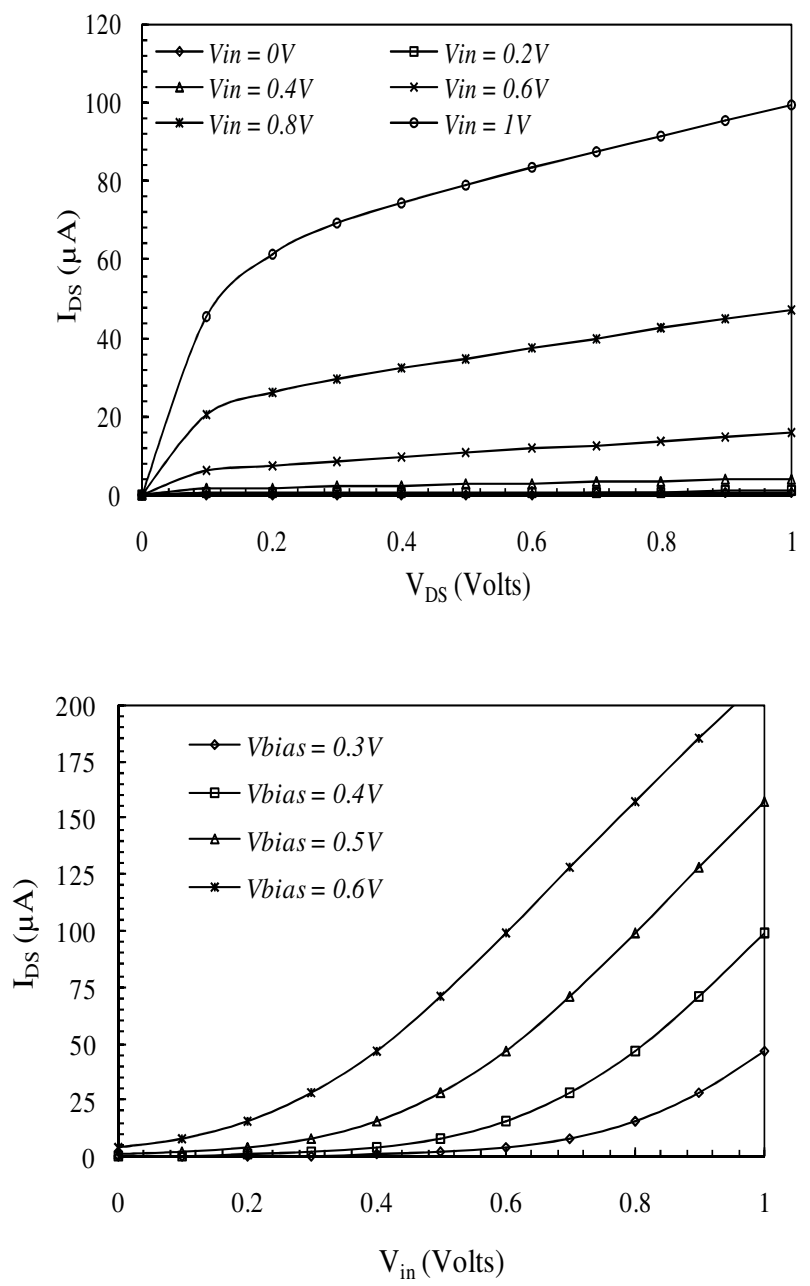

It has been observed that as we increase $V_{\text {bias }}$ from $0.3 \mathrm{~V}$ to $0.6 \mathrm{~V}$, effective threshold voltage $\left(V_{T, e f f}\right)$ of n-channel FGMOS decreases from $0.8 \mathrm{~V}$ to $0.2 \mathrm{~V}$ at a reference drain current of $20 \mu \mathrm{A}$. Similarly, the drain and transfer characteristics for p-channel FGMOS show bias dependent behaviour. Thus, the performance of n-channel and p-channel FGMOS can be varied by optimum selection of their respective $V_{\text {bias }}$ and making FGMOS based digital circuits suitable for low voltage and low power applications.

\section{FGMOS INVERTER}

The architecture of the FGMOS inverter has been obtained from the conventional CMOS inverter as shown in Fig. 4 [13]. The bias voltages $V_{b p}$ and $V_{b n}$ provide tunability to the threshold voltages of M1 and M2 respectively. It is, therefore, expected that by varying the bias voltages, the threshold voltage of the FGMOS inverter can be changed. 


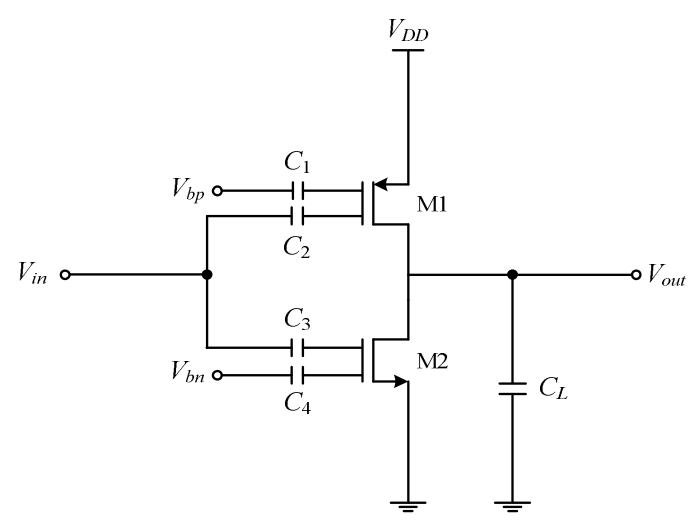

Figure 4. FGMOS inverter

The performance of FGMOS inverter can be characterized through its voltage transfer characteristics (VTC) which is a plot of $V_{\text {out }}$ as a function of $V_{i n}$. From these characteristics we can calculate parameters like switching threshold voltage and noise margins to ascertain the dc performance of these circuits. The switching threshold voltage $\left(V_{S}\right)$ is defined as the input voltage that gives an identical output voltage and it can be obtained from the intersection of the VTC curve and the plot of $V_{\text {out }}=V_{\text {in }}[13,14]$. The voltage noise margins can be obtained as $N M_{H}=V_{O H}$ - $V_{S}$ and $N M_{L}=V_{S}-V_{O L}$, where $V_{O H}$ and $V_{O L}$ are logic-high and logic-low output voltages of inverter respectively. Since noise margins $N M_{H}$ and $N M_{L}$ account for the sensitivity of a gate to noise, therefore large value of this parameter is desired that makes the gate less sensitive to noisy environment [15].

The switching threshold voltage for FGMOS inverter is given by [14]:

$$
V_{S}=\frac{V_{D D}-\left|\frac{V_{T p}-V_{b p} k_{2}}{k_{1}}\right|+\sqrt{\frac{\beta_{\mathrm{n}}}{\beta_{\mathrm{p}}}}\left(\frac{V_{T n}-V_{b n} k_{2}}{k_{1}}\right)}{1+\sqrt{\frac{\beta_{\mathrm{n}}}{\beta_{\mathrm{p}}}}}
$$

where $\beta_{\mathrm{n}}=\mu_{n} C_{o x}\left(\frac{W}{L}\right)_{n}$ and $\beta_{\mathrm{p}}=\mu_{p} C_{o x}\left(\frac{W}{L}\right)_{p}$ are transconductance parameters of $\mathrm{n}$ and $\mathrm{p}$ channel FGMOS.

Now, the circuit of FGMOS inverter has been simulated to obtain the voltage transfer characteristics (VTC) at different values of $V_{b p}$ and $V_{b n}$ by selecting $W / L$ of M1 as $26 \mu \mathrm{m} / 0.13 \mu \mathrm{m}$ and $\mathrm{M} 2$ as $13 \mu \mathrm{m} / 0.13 \mu \mathrm{m}$ with a supply voltage of $1 \mathrm{~V}$ as shown in Figs. 5 and 6 respectively. 
Electrical and Electronics Engineering: An International Journal (ELELIJ) Vol 5, No 2, May 2016

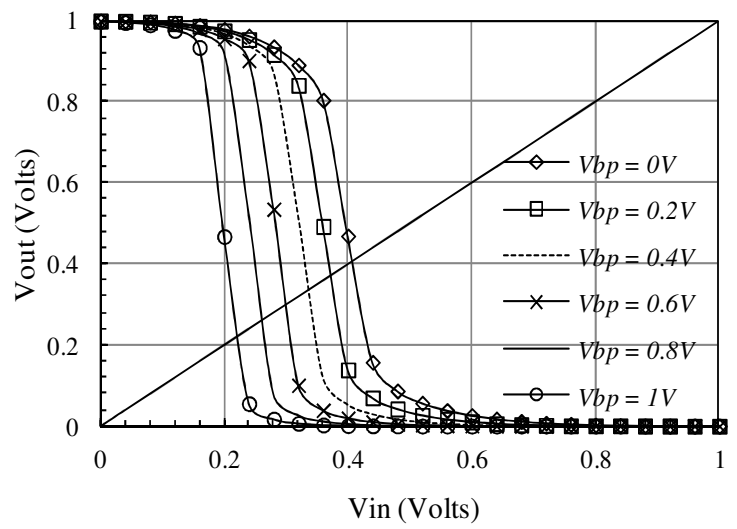

Figure 5. VTC of FGMOS inverter at different $V_{b p}$

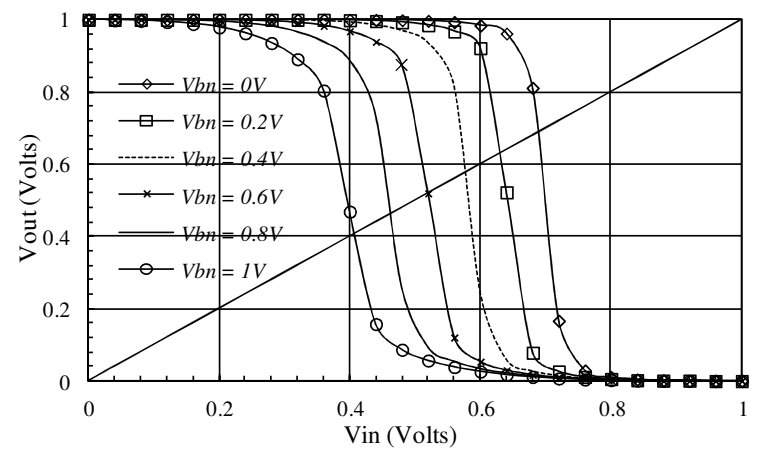

Figure 6. VTC of FGMOS inverter at different $V_{b n}$

From Figs. 5 and 6, the calculated values of the switching threshold voltage $\left(V_{S}\right)$ and noise margins $N M_{H}$ and $N M_{L}$ at different values of $V_{b p}$ and $V_{b n}$ are given in table 1.

Table 1. Noise margins at different $V_{b p}$ and $V_{b n}$

\begin{tabular}{|c|c|c|c|c|c|c|c|}
\hline \multicolumn{5}{|c|}{$V_{b n}=1$ V fixed } & \multicolumn{4}{c|}{$V_{b p}=0$ V fixed } \\
\hline $\begin{array}{c}V_{b p} \\
\text { (Volts) }\end{array}$ & $\begin{array}{c}V_{S} \\
(\text { Volts })\end{array}$ & $\begin{array}{c}N M_{H} \\
(\text { Volts) }\end{array}$ & $\begin{array}{c}N M_{L} \\
(\text { Volts })\end{array}$ & $\begin{array}{c}V_{b n} \\
(\text { Volts) }\end{array}$ & $\begin{array}{c}V_{S} \\
(\text { Volts) }\end{array}$ & $\begin{array}{c}N M_{H} \\
\text { (Volts) }\end{array}$ & $\begin{array}{c}N M_{L} \\
\text { (Volts) }\end{array}$ \\
\hline 0 & 0.40 & 0.60 & 0.40 & 0 & 0.68 & 0.32 & 0.68 \\
\hline 0.2 & 0.37 & 0.63 & 0.37 & 0.2 & 0.63 & 0.37 & 0.63 \\
\hline 0.4 & 0.33 & 0.67 & 0.33 & 0.4 & 0.57 & 0.43 & 0.57 \\
\hline 0.6 & 0.29 & 0.71 & 0.29 & 0.6 & 0.52 & 0.48 & 0.52 \\
\hline 0.8 & 0.25 & 0.75 & 0.25 & 0.8 & 0.46 & 0.54 & 0.46 \\
\hline 1 & 0.21 & 0.79 & 0.21 & 1 & 0.40 & 0.60 & 0.40 \\
\hline
\end{tabular}

As observed in table 1, when bias voltage of p-channel FGMOS $\left(V_{b p}\right)$ is varied from $0 \mathrm{~V}$ to $1 \mathrm{~V}$ at constant bias voltage of n-channel FGMOS $\left(V_{b n}=1 \mathrm{~V}\right)$, the switching threshold voltage $\left(V_{S}\right)$ and low noise margin $\left(N M_{L}\right)$ of FGMOS inverter decreases from $0.40 \mathrm{~V}$ to $0.21 \mathrm{~V}$ but high noise 
margin $\left(N M_{H}\right)$ increases from $0.60 \mathrm{~V}$ to $0.79 \mathrm{~V}$. Similarly, when bias voltage of n-channel FGMOS $\left(V_{b n}\right)$ is increased from $0 \mathrm{~V}$ to $1 \mathrm{~V}$, while keeping $V_{b p}$ fixed at $0 \mathrm{~V}, N M_{L}$ decreases from $0.68 \mathrm{~V}$ to $0.40 \mathrm{~V}$. Now, in the circuit of FGMOS inverter, M2 (n-channel) determines $N M_{L}$ and M1 (p-channel) determines $N M_{H}$ and both noise margins are desired to be high for better noise immunity. From table 1, we observe that $N M_{H}$ and $N M_{L}$ are maximum when $V_{b p}=0 \mathrm{~V} \& V_{b n}=1$ $\mathrm{V}$ which is the condition of low voltage operation for FGMOS because for $V_{b p}=0 \mathrm{~V} \& V_{b n}=1 \mathrm{~V}$, M1 and M2 exhibits minimum value of threshold voltage. Therefore, better noise margins can be optimized by appropriate selection of $V_{b p}$ and $V_{b n}$ at $0 \mathrm{~V}$ and $1 \mathrm{~V}$ respectively.

The transient behaviour of the FGMOS inverter can be characterized by propagation delay $\left(t_{p}\right)$ which is defined as the average of the time delay from low-to-high transition $\left(t_{p l h}\right)$ and from highto-low transition $\left(t_{p h l}\right)$ of the input and output waveforms in an inverter. It specifies the operating speed and is given as $[15,16]$ :

$t_{p}=\frac{\left(t_{p l h}+t_{p h l}\right)}{2}$

The propagation delay for FGMOS inverter can be given as [14]:

$t_{p}=0.35 C_{L}\left\{\left[\beta_{\mathrm{n}}\left(V_{D D}-\frac{\left(V_{T n}-V_{b n} k_{2}\right)}{k_{1}}\right)\right]^{-1}+\left[\beta_{\mathrm{p}}\left(V_{D D}-\frac{\left(V_{T p}-V_{b p} k_{2}\right)}{k_{1}}\right)\right]^{-1}\right\}$

Since $t_{p}$ depends on threshold voltage of $\mathrm{n}$ and p-channel MOSFETs, therefore it is expected that it can be optimized using FGMOS where threshold voltage tunability is feasible [12].

The transient characteristics of FGMOS inverter at different values of $V_{b p}$ and $V_{b n}$ are shown in Figs. 7 and 8 respectively.

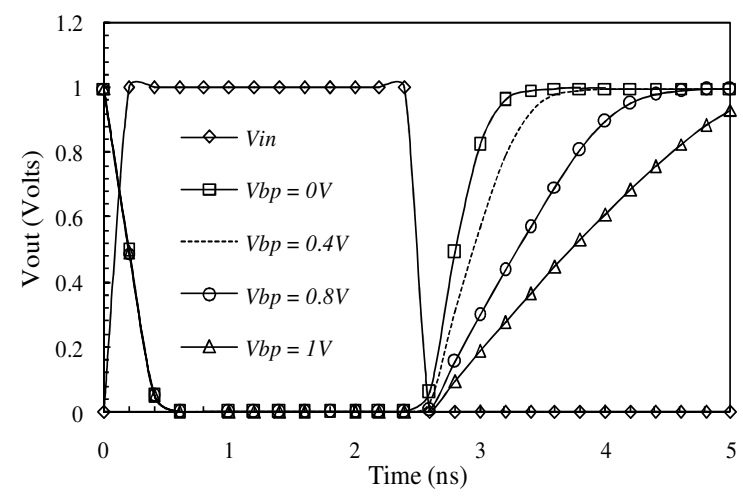

Figure 7. Transient response of FGMOS inverter at different $V_{b p}$ 
Electrical and Electronics Engineering: An International Journal (ELELIJ) Vol 5, No 2, May 2016

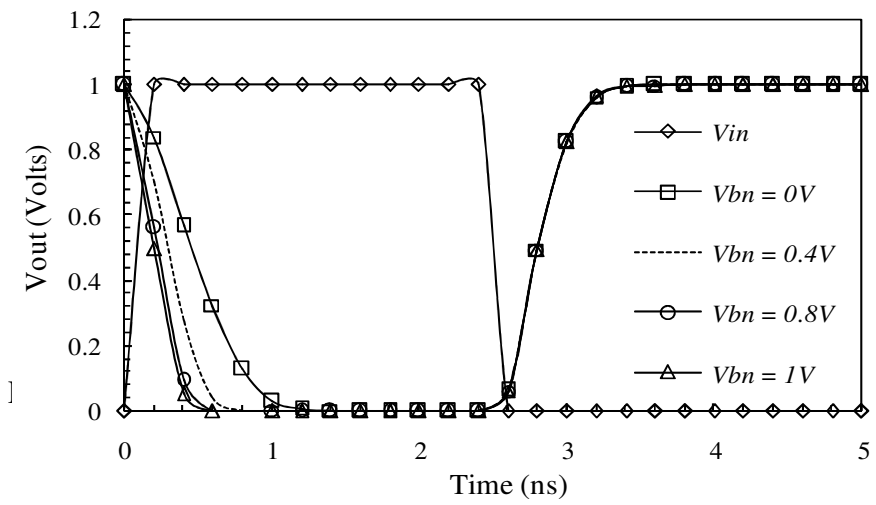

It is seen that the pulse response of FGMOS inverter can be varied with bias voltage resulting in different values of propagation delay as shown in Fig. 9.

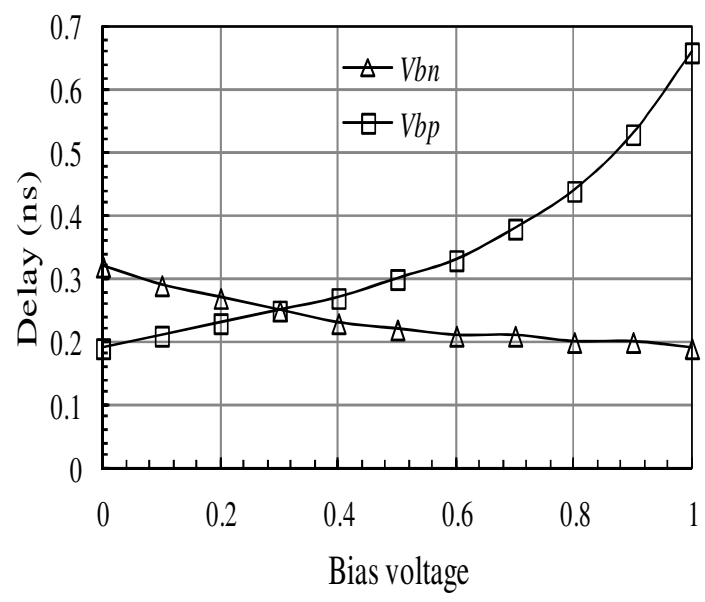

Figure 9. Propagation delay at different bias voltage

It has been observed in Fig. 9 that if the bias voltage of p-channel FGMOS is increased from $0 \mathrm{~V}$ to $1 \mathrm{~V}$ while keeping bias voltage of $\mathrm{n}$-channel FGMOS fixed at $1 \mathrm{~V}$, the propagation delay increases from 0.19 ns to 0.66 ns. Similarly, if bias voltage of n-channel FGMOS is increased from $0 \mathrm{~V}$ to $1 \mathrm{~V}$ while keeping bias voltage of p-channel FGMOS fixed at $0 \mathrm{~V}$, the propagation delay decreases from $0.32 \mathrm{~ns}$ to $0.19 \mathrm{~ns}$. Therefore, the appropriate selection of bias voltages of $n$ and p-channel FGMOS at $1 \mathrm{~V}$ and $0 \mathrm{~V}$ respectively decreases the propagation delay of FGMOS inverter, thus enhancing the operating speed.

Now, the comparative transient characteristics of CMOS and FGMOS inverters have been obtained by selecting $V_{b p}=0 \mathrm{~V}$ and $V_{b n}=1 \mathrm{~V}$ as shown in Fig. 10. 
Electrical and Electronics Engineering: An International Journal (ELELIJ) Vol 5, No 2, May 2016

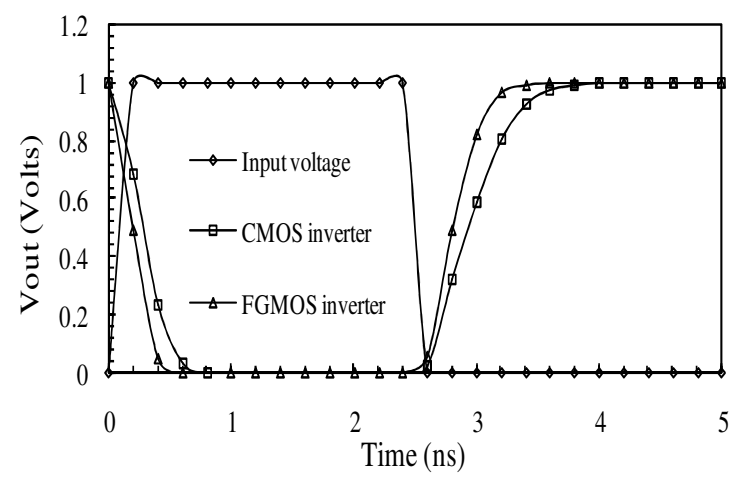

Figure 10. Comparative transient characteristics of CMOS and FGMOS inverters

From these results, it has been observed that FGMOS based inverter has less propagation delay $(0.2 \mathrm{~ns})$ as compared to CMOS inverter which has the propagation delay of $0.4 \mathrm{~ns}$, implying that FGMOS based inverter exhibits better switching response and has high operating speed.

Since, the energy delay product represents the trade-off between power dissipation and the speed, implying the operation of digital circuits at low power would result in loss of speed. Therefore, lower value of energy delay product is required for circuits suitable for operation with low operating voltage and low power consumption without much loss in operating speed. Now, the values of propagation delay obtained from the transient analysis of CMOS and FGMOS based inverters has been used to calculate the energy delay product (EDP) at different values of $V_{D D}$ as shown in Fig. 11.

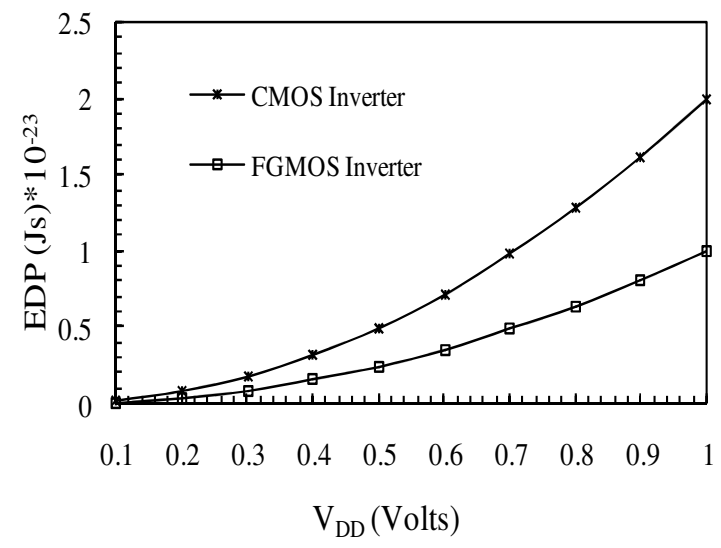

Figure 11. Comparative EDPs of CMOS and FGMOS inverters

The results obtained from Fig. 11 show that EDP is a function of supply voltage and for $V_{D D}=1 \mathrm{~V}$, FGMOS inverter has EDP of $1 \times 10^{-23} \mathrm{Js}$ where as the value of EDP for CMOS inverter is $2 \times 10^{-23}$ Js. Therefore, FGMOS inverter shows better performance as compared to CMOS due to lower value of energy delay product, thus posing as an alternative design technique for low voltage and high speed digital circuits. 
Electrical and Electronics Engineering: An International Journal (ELELIJ) Vol 5, No 2, May 2016

\section{Ring OSCILlator}

Since FGMOS inverter exhibits better response than its CMOS counterpart in terms of speed, noise immunity and power dissipation, therefore it has been further employed to implement a ring oscillator which is often used in the information, communication and sensor technology for frequency translation and channel selection [17-19].

A ring oscillator based on FGMOS is shown in Fig. 12 which consists of a cascade of three inverters and the frequency of oscillation is given by [20]:

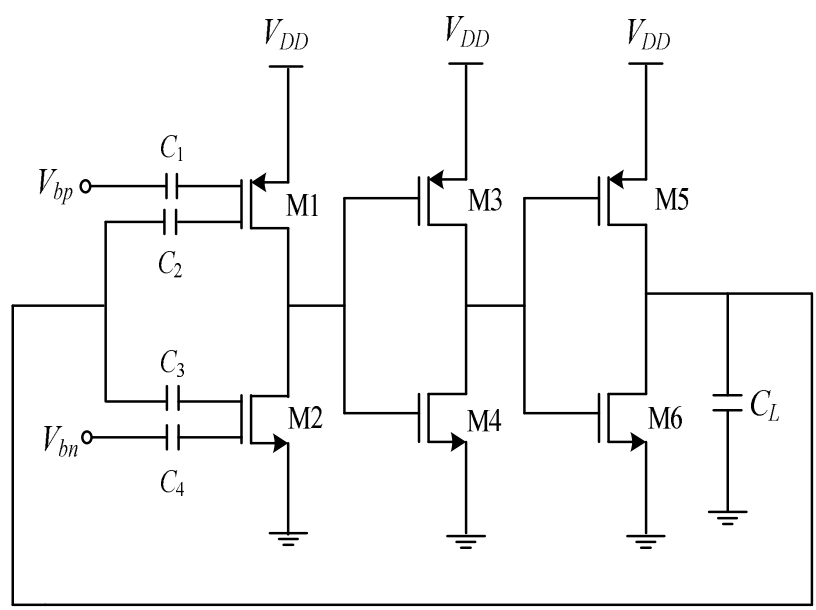

$f_{o}=\frac{1}{6\left(t_{p h l}+t_{p l h}\right)}$

Figure 12. FGMOS based ring oscillator

where $t_{p h l}$ and $t_{p l h}$ are the propagation delay for high-to-low and low-to-high transitions respectively in an inverter which are given as under:

$$
\begin{aligned}
& t_{p h l}=\frac{C_{L} k_{1}\left(k_{1} V_{D D}+V_{T n}-V_{b n} k_{2}\right)}{\beta_{n}\left(k_{1} V_{D D}-V_{T n}+V_{b n} k_{2}\right)^{2}} \\
& t_{p l h}=\frac{C_{L} k_{1}\left\{k_{1} V_{D D}+3\left(V_{T p}-V_{b p} k_{2}\right)\right\}}{\beta_{p}\left(k_{1} V_{D D}+V_{T p}-V_{b p} k_{2}\right)^{2}} \\
& t_{p h l}+t_{p l h}=C_{L} k_{1}\left(\frac{k_{1} V_{D D}+V_{T n}-V_{b n} k_{2}}{\beta_{n}\left(k_{1} V_{D D}-V_{T n}+V_{b n} k_{2}\right)^{2}}+\frac{k_{1} V_{D D}+3\left(V_{T p}-V_{b p} k_{2}\right)}{\beta_{p}\left(k_{1} V_{D D}+V_{T p}-V_{b p} k_{2}\right)^{2}}\right)
\end{aligned}
$$


Electrical and Electronics Engineering: An International Journal (ELELIJ) Vol 5, No 2, May 2016

From Eq. 8, we see that propagation delay decreases with increase in $V_{b n}$ and decrease in $V_{b p}$. Therefore, increasing bias voltage of n-channel FGMOS while keeping bias voltage of p-channel FGMOS at zero volt will lead to small propagation delay and hence, enhanced frequency of oscillation. Now, the circuit of FGMOS ring oscillator has been simulated at different values of $V_{b n}$ while keeping $V_{b p}$ fixed at $0 \mathrm{~V}$ by selecting $W / L$ of M1, M3 and M5 as $26 \mu \mathrm{m} / 0.13 \mu \mathrm{m}$ and M2, M4 and M6 as $13 \mu \mathrm{m} / 0.13 \mu \mathrm{m}$ with the supply voltage of $1 \mathrm{~V}$. The simulation results are shown in Fig. 13.

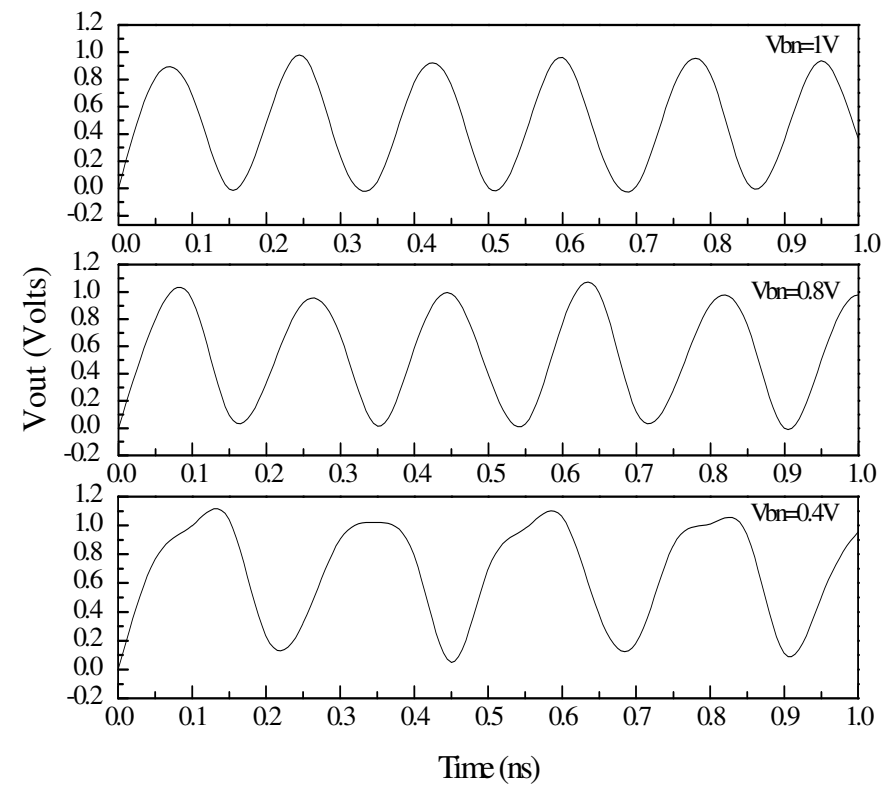

Figure 13. Oscillations of FGMOS based ring oscillator at different $V_{b n}$

From the above results we have observed that the frequency of oscillation increases with increase in bias voltage $\left(V_{b n}\right)$ as shown in table 2 .

Table 2. Variation of $f_{o}$ with $V_{b n}$

\begin{tabular}{|c|c|}
\hline $\boldsymbol{V}_{\boldsymbol{b n}}(\mathbf{V})$ & $\boldsymbol{f}_{\boldsymbol{o}}(\mathbf{G H z})$ \\
\hline 0.2 & 4 \\
\hline 0.4 & 4.5 \\
\hline 0.6 & 5.2 \\
\hline 0.8 & 5.9 \\
\hline 1 & 6.7 \\
\hline
\end{tabular}


The comparative performance of CMOS and FGMOS based three stage ring oscillator has been obtained by selecting $V_{b p}=0 \mathrm{~V}$ and $V_{b n}=1 \mathrm{~V}$ and is shown in Fig. 14. It has been observed that frequency of oscillations in FGMOS ring oscillator is $6.7 \mathrm{GHz}$ while for CMOS ring oscillator it is $5 \mathrm{GHz}$.

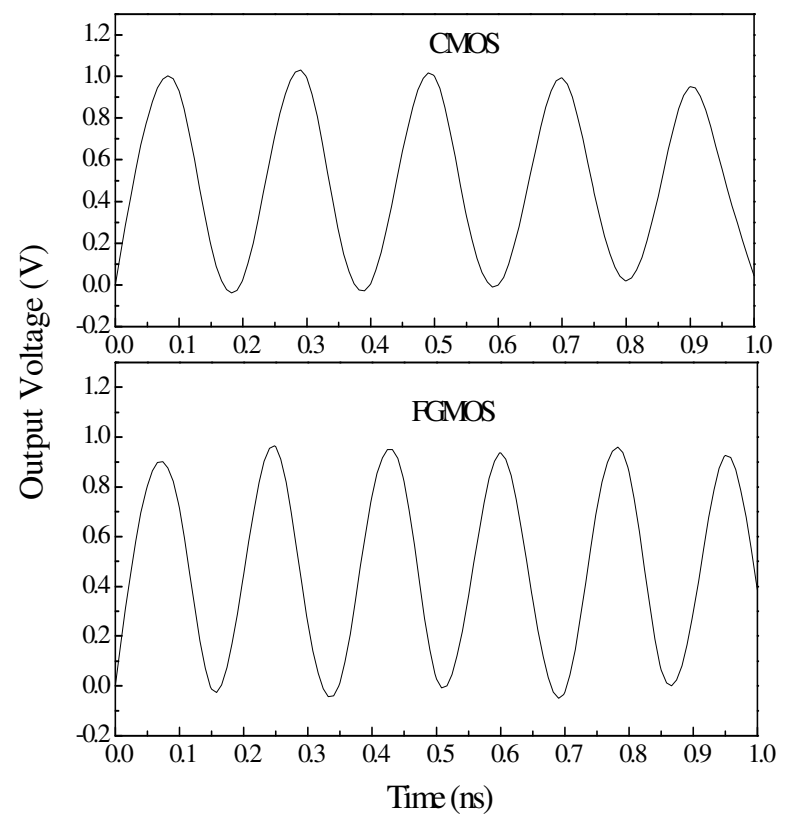

Figure 14. Oscillations of CMOS and FGMOS based ring oscillators

The number of inverter stages in ring oscillator may be increased for multiphase outputs but at the expense of reduced operating speed, high power dissipation and large chip area [20]. The comparative frequency of oscillations in ring oscillator using CMOS and FGMOS with different stages is given in table 3

\begin{tabular}{|c|c|c|}
\hline \multirow{2}{*}{$\begin{array}{c}\text { No. of } \\
\text { stages }\end{array}$} & CMOS & FGMOS \\
\cline { 2 - 3 } & $\boldsymbol{f}_{\boldsymbol{o}}(\mathbf{G H z})$ & $\boldsymbol{f}_{\boldsymbol{o}}(\mathbf{G H z})$ \\
\hline 3 & 5 & 6.7 \\
\hline 5 & 3.3 & 3.8 \\
\hline 7 & 2.5 & 2.8 \\
\hline 9 & 2 & 2.1 \\
\hline
\end{tabular}

Table 3. Comparative oscillation frequency of multistage ring oscillator

From the above results, we observe that with increase in the number of inverter stages in the structure of ring oscillator, the frequency of oscillation decreases due to increased propagation delay. 
Electrical and Electronics Engineering: An International Journal (ELELIJ) Vol 5, No 2, May 2016

\section{Conclusions}

In this paper, we have studied the effect of threshold voltage tunability in FGMOS for enhancing the performance of inverter and ring oscillator. It has been found that by varying the bias voltage of FGMOS, the voltage transfer characteristics of inverter can be suitably altered resulting in decreased switching threshold voltage, increased noise margins, reduced propagation delay and energy delay product as compared to its CMOS version. Further, it has been observed that the oscillation frequency of ring oscillator depends on the propagation delay of inverter stages and FGMOS based ring oscillator exhibits higher frequency of oscillation as compared to CMOS ring oscillator.

\section{REFERENCES}

[1] A. P. Chandrakasan, S. Sheng \& R. W. Brodersen, (1992) "Low power CMOS digital design", IEEE J. Solid-State Circuits, Vol. 27, No. 4, pp. 473-484.

[2] R. Gonzalez, B. M. Gordon \& M. A. Horowitz, (1997) "Supply and threshold voltage scaling for low power CMOS”, IEEE J. Solid-State Circuits, Vol. 32, No. 8, pp. 1210-1216.

[3] E. S. Sinencio \& A. G. Andreou, (1997) "Low-Voltage/Low-Power Integrated Circuits and Systems", IEEE Press.

[4] S. Yan \& E. S. Sinencio, (2000) "Low Voltage Analog Circuit Design Techniques: A Tutorial", IEICE Trans. Analog Integrated Circuits and Systems, Vol. E00-A, No. 2, pp. 1-17.

[5] C. J. B. Fayomi, M. Sawan \& G. W. Roberts, (2004) "Reliable Circuit Techniques for Low Voltage Analog Design in Deep Sub micron Standard CMOS: A Tutorial”, Analog Integr. Circuits Signal Proc., Vol. 39, No.1, pp. 21-38.

[6] J. R. Angulo, S. C. Choi \& G. G. Altamirano, (1995) "Low voltage circuits building blocks using multiple input floating gate transistors", IEEE Trans. Circuits Syst.-I, Vol. 42, No. 11, pp. 971-974.

[7] J. R. Angulo, G. G. Altamirano \& S. C. Choi, (1997) "Modeling multiple-input floating-gate transistors for analog signal processing", Proc. IEEE Int. Symp. Circuits Syst., Vol. 3, pp. 2020-2023.

[8] P. Hasler \& T. S. Lande, (2001) "Overview of floating-gate devices, circuits and systems", IEEE Transactions on Circuits and Systems - II: Analog and Digital Signal Processing, Vol.48, No.1, pp. 13.

[9] E. R. Villegas, (2006) Low Power and Low Voltage Circuit Design with FGMOS Transistor, IET Circuits, Devices and Systems, Series 20.

[10] M. Gupta \& R. Pandey, (2010) "FGMOS based voltage-controlled resistor and its applications", Microelectronics Journal, Vol. 41, pp. 25-32.

[11] A. Kumar, (2013) "Split length FGMOS MOS cell: a new block for low voltage applications", Analog Integrated Circuits and Signal Processing, Vol. 75, pp. 399-405.

[12] S. Sharma, S. S. Rajput, L. K. Mangotra \& S. S. Jamuar, (2006) "FGMOS current mirror: behavior and bandwidth enhancement", Analog Integrated Circuits and Signal Processing, Vol. 46 pp. 281286.

[13] J. Rabaey, A. Chandrakasan \& B. Nikolic, (2003) Digital Integrated circuits, A Design Perspective, Prentice Hall.

[14] J. P. Uyemura, (2002) Introduction to VLSI circuits and systems, John Wiley and sons, New Delhi.

[15] K. Martin, (2000) Digital Integrated circuit design, Oxford university press.

[16] B. Razavi, (2008) Fundamentals of Microelectronics, John Wiley and sons.

[17] C. H. Park, O. Kim \& B. Kim, "A 1.8-GHz self-calibrated phase locked loop with precise I/Q matching”, IEEE J. Solid-State Circuits, Vol. 36, pp. 777-783, 2001.

[18] J. Savoj \& B. Razavi, (2001) "A 10-Gb/s CMOS clock and data recovery circuit with a half-rate linear phase detector", IEEE J. Solid-State Circuits, Vol. 36, pp. 761-767.

[19] M. Alioto \& G. Palumbo, (2001) "Oscillation frequency in CML and ESCL ring oscillators", IEEE Trans. Circuits Syst. I, Vol. 48, pp. 210-214.

[20] M. K. Mandal \& B. C. Sarkar, (2010) "Ring oscillators: characteristics and applications", Indian journal of Pure and Applied Physics, Vol. 48, pp. 136-145. 
Electrical and Electronics Engineering: An International Journal (ELELIJ) Vol 5, No 2, May 2016

\section{AUTHORS}

Susheel Sharma received his MSc Physics (Electronics) from University of Jammu, Jammu, India in 1991 and PhD degree from the same University in 2007 on the title 'Analog applications of Floating-Gate MOS transistor in current mirrors and current conveyors'. He has been working as faculty in the Department of Physics \& Electronics, University of Jammu since 1995. Presently he is an Associate Professor in Electronics and his area of research interest includes Low voltage analog and digital integrated circuits and he has 44 publications to his

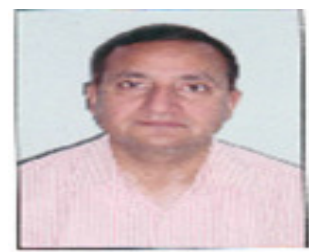
credit in various National/International conferences and journals. He is a life member of Institution of Electronics and Telecommunication Engineers (IETE) India and Indian Science Congress.

Rockey Gupta received his M.Sc. degree in Electronics from University of Jammu in 2000 with gold medal. He received his Ph. D degree in Electronics in 2014 from the same university. He has been working as a faculty member in the department of Physics and Electronics since March 2002. He has been teaching courses of electronic devices and circuits, digital electronics, IC technology, microprocessors and microcontrollers and computer programming with c++ to M.Sc electronics students. He is a life member of Institution of Electronics and

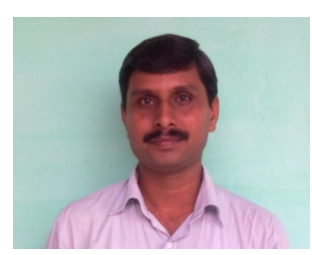
Telecommunication Engineers (IETE) India and Indian Science Congress. Presently he is working as a senior Assistant Professor in Department of Physics and Electronics, University of Jammu. His area of research includes low voltage analog circuit design techiques using quasi-flaoting-gate MOSFETs. He has 20 publications to his credit in various National/International conferences and journals.

Roshani Gupta received her M.Sc. and M.Phil. in Electronics from University of Jammu in the years 2011 and 2014, respectively. Presently she is pursuing Ph.D. in Electronics from the same University. Her research interests include designing CMOS digital circuits for low power applications.

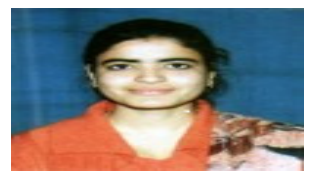

